\title{
Une Géo-politique de la Regio Genevensis
}

Thème nouveau de recherche, la coopération transfrontalière donne lieu, ces dernières années, à une abondante littérature. L'émergence de liaisons pardessus les frontières des Etats-nations suscite l'imagination: le temps de la division, des découpages administratifs serait-il fini pour faire place à celui de la création de nouvelles unités territoriales transfrontalières et internationales correspondant aux espaces vécus par les populations? On peut y croire, puisque bien des problèmes contemporains, écologiques, économiques et énergétiques doivent être abordés à l'échelle internationale. Mais si les liens entre les régions frontalières se renforcent, il ne faut pas oublier que les Etats sont rarement disposés à céder des éléments de leur souveraineté nationale...

La région franco-genevoise constitue pourtant une exception dans ce domaine: non seulement elle a été pionnière en Europe pour établir une Commission Consultative transfrontalière bipartite acceptée par les gouvernements (1973), mais de plus, elle est la première à "passer outre à l'un des tabous de la souveraineté nationale absolue. C'est en effet à l'initiative du Gouvernement de la République et Canton de Genève qu'une part importante des impôts payés à Genève par les travailleurs frontaliers se trouve reversée aux communes françaises de résidence par l'Etat de Genève» (DE ROUGEMENT). En renonçant à conserver une partie de l'impôt régulier, l'Etat de Genève ouvre une porte majeure à la coopération transfrontalière; la réalité physique favorisait ce mouvement que la tradition culturelle et étatique freinait. A travers le cas de la région francogenevoise, cet article va analyser comment le fonctionnement de l'Etat-nation, qui ne correspond plus aux réalités du XXe siècle, est progressivement mis en cause par le renforcement des liaisons transfrontalières et internationales.

Bien souvent la Regio Genevensis sert d'exemple dans les relations transfrontalières; position enviable, mais aussi difficile, car l'engagement international d'un canton non souverain face à un Etat centralisé résulte de compromis bien fragiles et délicats à élaborer. En effet les mesures internationales franco-genevoises dépendent de relations triangulaires entre trois acteurs: un canton non souverain, la République et Canton de Genève, le gouverne- ment fédéral suisse et l'Etat français. Depuis peu un quatrième partenaire, non souverain, la région Rhône-Alpes, apparaît dans ce jeu transnational. Guidé par des intérêts spécifiques chaque acteur propose des séries d'actions pour faciliter (ou entraver) le fonctionnement régional transfrontalier. Ces intérêts sont souvent de nature pragmatique, liés aux pratiques de populations qui désirent vivre dans une région frontière. Notre recherche repose sur l'hypothèse que les actions transfrontalières résultent de pratiques territoriales; suite à l'apparition de relations quotidiennes transfrontalières les gouvernements doivent progressivement reconsidérer le rôle de la frontière. Notre analyse des relations transfrontalières doit donc partir d'une description des territorialités transnationales et des images mentales qui en résultent. Dans un deuxième temps alors nous pourrons voir comment ces pratiques territoriales génèrent la mise en place de politiques transfrontalières. La question est alors de savoir si ces mesures sont simplement locales ou si elles dépassent parfois ce niveau pour déboucher sur de véritables stratégies géo-politiques. Notre deuxième hypothèse est que la République et Canton de Genève possède une politique étrangère implicite, à l'échelle géographique régionale. Comme un Etat-nation, même s'il n'est pas souverain, le Canton de Genève cherche à régler ses problèmes frontaliers face à un Etat français qui n'a pas de stratégie précise à ce même niveau. D'où une ambiguïté dans les rapports entre deux Etats qui ne possèdent pas les mêmes pouvoirs et dont les engagements internationaux se situent à des échelles géographiques différentes. Les choix géo-politiques évoluent alors suivant les poids et les stratégies des divers acteurs régionaux et nationaux, comme en témoignent les mesures prises pour faciliter le fonctionnement de la Regio Genevensis.

Antoine S. Bailly, Prof., Université de Genève, Dept. de Géographie, 7, route de Drize, Case postale 266, 1227 Carouge 


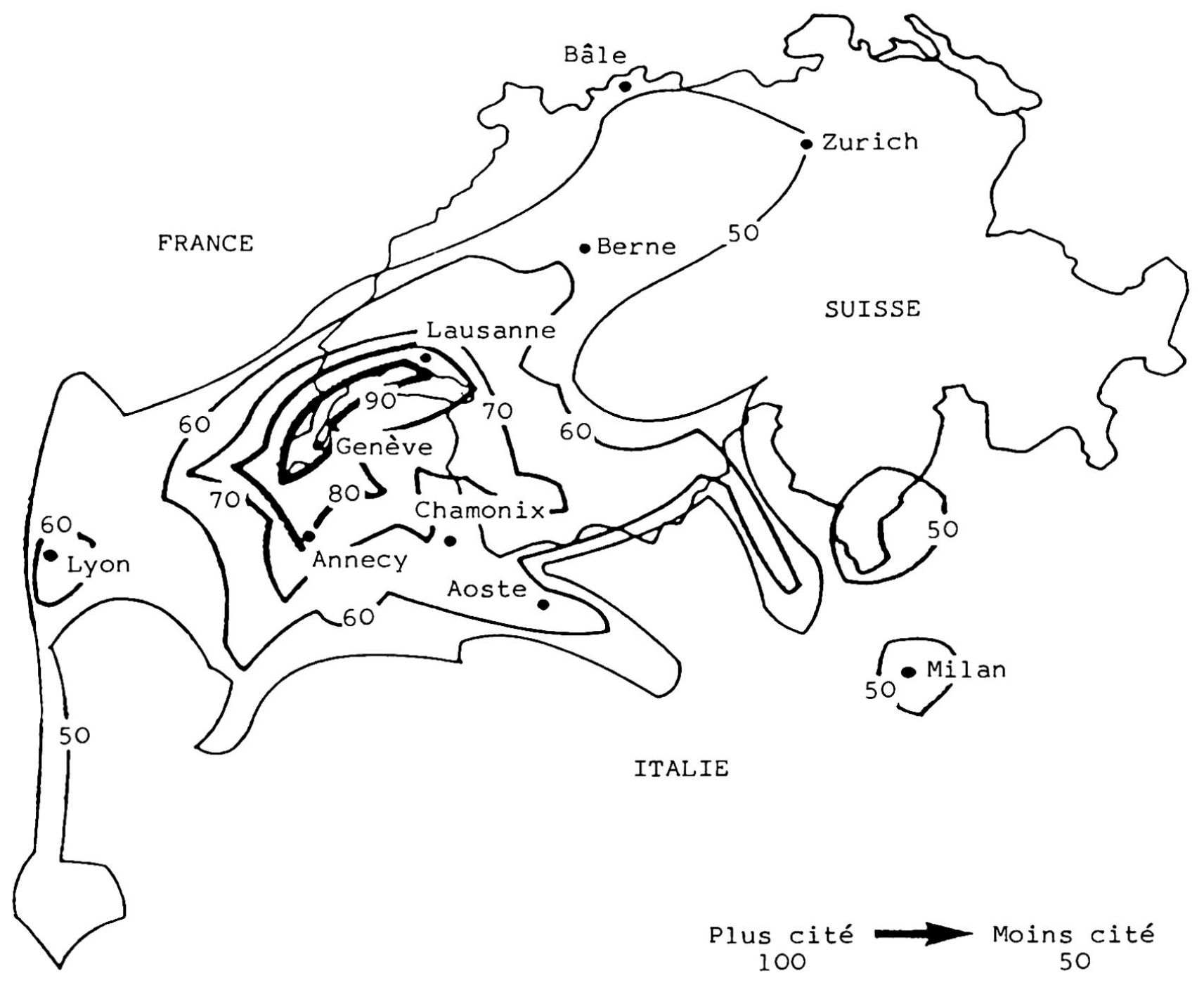

Fig. 1 Carte mentale des étudiants genevois (Enquête A. Bailly 1982)

\section{La région franco-genevoise: son image transfrontalière et internationale}

A l'exception d'un bassin naturel entre Jura et Salève, rien ne destinait Genève à devenir la métropole d'une région transfrontalière; l'histoire, la religion depuis 1536 (Réforme) séparait Genève de ses voisins de Savoie et du Pays de Gex. On imagine d'ailleurs souvent cette ville citadelle de la Réforme, enserrée dans ses frontières étroites indépendantes du milieu physique: celles-ci constituent en effet des limites qui ont marqué toute la vie de la République et Canton de Genève par les guerres qu'elles ont suscitées et par les traités qui en ont défini les tracés.

Et pourtant, comme le montre VERNEX (1981), l'image actuelle de Genève s'articule autour de trois pôles, dont deux sont d'essence internationale:
- la ville de la Réforme

- la métropole lémanique, active, internationale, au cadre naturel prestigieux

- la ville intellectuelle

La diffusion de cette triple image concourt à élargir la renommée de la ville au-delà de son territoire propre, et l'image finit par prendre le pas sur le réel, à

- Une étude menée en 1986 par l'Institut IPSO, Sozial- und Umfrageforschung de Zurich dans l'ensemble de la Suisse confirme cette identité propre des Genevois; selon ce sondage les Genevois ne se passionnent guère pour ce qui se passe dans le reste de la Suisse, connaissent mal leurs Confédérés et sont au contraire tournés vers la France. En résulte une image de Genève dans les autres cantons qui, sans être mauvaise, est incomplète et floue. 
estomper les frontières, à faire naître un fonctionnement international au-dessus des Etats...

Il n'est donc pas étonnant de constater, lorsque l'on interroge des résidents sur leur image de Genève, de voir disparaître les frontières; apparaît par contre une métropole internationale avec ses quartiers différenciés, son palais des Expositions, ses monuments (jet d'eau, cathédrale), son aéroport et sa zone agricole, de loisirs et de résidences secondaires qui débordent largement les frontières. Ainsi la Figure no 1, carte mentale d'étudiants genevois, montre-t-elle très clairement une région internationale franco-genevoise. Elle correspond à la fois à un espace perçu et à un espace vécu. C'est en fonction de leurs espaces-activités que les Genevois interrogés se représentent leur région. Si l'effet frontière est très marqué vers le Nord-Ouest, c'est suite à la barrière physique des montagnes du Jura et non aux limites internationales; la territorialité genevoise déborde sur le Pays de Gex et encore plus sur la Savoie, aussi fréquentée que le Canton de Vaud. L'isoligne 70 , témoignant d'une forte fréquentation, s'étend plus sur la France, au Sud de Genève, que sur la Suisse. Ajoutons même que la capitale fédérale, Berne, est moins souvent mentionnée que Lyon et Aoste: l'aire représentée sur les cartes mentales témoigne de la puissance de la francophonie et du rôle des limites linguistiques et culturelles.

L'organisation de ce territoire franco-genevois s'articule à partir des grands axes naturels que constituent les vallées de l'Arve, du Rhône, les bords du Lac Léman, les vallées alpines et les voies de communication qui y ont été créées. En ce sens la proximité du nœud autoroutier français (vers le tunnel du Mont-Blanc, Lyon-Grenoble, Mâcon), aux frontières du Canton de Genève, n'est pas sans influence sur l'élargissement de la territorialité genevoise: la réduction des distances-temps permet une extension des pratiques spatiales, en particulier pour les petites vacances. Ce territoire est organisé dans l'image mentale des Genevois, à partir de repères proches comme le Salève, le Môle, les Voirons, le Jura, tous situés en France, et plus lointains comme le massif du Mont-Blanc, les Préalpes du Chablais, la vallée du Rhône en aval de Lyon, et même la plaine de Lombardie. Les limites de ce territoire, en expansion, restent cependant très floues, fonction des vécus personnels et on ne peut les généraliser. Ainsi constate-t-on l'intégration de Genève à un double espace, l'un étroit, celui de la Suisse romande, et l'autre plus vaste et plus flou, celui de la Francophonie. Un territoire de loisirs, reflétant les valeurs d'une société mobile, déborde du centre fonctionnel que constituent la ville de Genève et dans une moindre mesure le canton et les zones frontalières proches. On peut parler, dans ce cas, de dualité, de complémentarité et d'intégration spatiale. Dualité de deux espaces séparés administrativement et présentant des caractères économiques et socio-politiques différents, complémentarité de leurs activités, mais intégration des espaces vécus et des pratiques.

Après avoir mené une deuxième enquête sur les cartes mentales des frontaliers français (en provenance de Savoie et du Pays de Gex, fig. no 2), nous constatons de nombreuses similitudes d'ensemble: centrées également sur Genève, elles sont pourtant moins orientées vers la Suisse et plus vers certaines capitales régionales françaises (Lyon, Grenoble, Bourg-en-Bresse). Dualité entre le lieu de travail et celui de résidence implanté dans la structure administrative française, mais intégration à une grande région franco-genevoise, marquent cette image; la zone d'habitat-travail peut ainsi être assimilée à celle du grand Genève, même si la frontière coupe officiellement l'agglomération en deux ensembles nationaux et si les pratiques culturelles divergent, comme en témoigne l'analyse de la presse. Ainsi la Tribune, ou le Dauphiné n'ont-ils chacun que de maigres rubriques concernant le pays voisin. L'emphase est mise sur les tendances nationales. Seraitce à dire que l'espace franco-genevois existe malgré le carcan des structures nationales? Comme souvent les pratiques se vivent avant que les institutions n'en tiennent compte... Une analyse des cartes mentales des écoliers de St-Julien (France) montre l'absence de la frontière dans les images des enfants; elle ne constitue plus ni une limite ni un repère spatial (enquête de M. André, professeur au Lycée de St-Julien) pour les plus jeunes. Serait-ce une tendance pour l'avenir?

\section{Des flux migratoires transfrontaliers et transnationaux}

\subsection{Les frontaliers: travailler en Suisse, vivre en France}

On oublie souvent que, comme la question d'image transfrontalière, l'étude du phénomène des travailleurs frontaliers est récente. Nulle pendant la guerre et peu nombreuse auparavant (et surtout dans sens Suisse-France) la main d'œuvre frontalière française travaillant dans le Canton de Genève atteint 6000 personnes en 1965, 10000 en 1968, 20000 en 1972 pour culminer à 25000 en 1974. Avec la récession économique ce chiffre tombe à 18000 en 1977 pour remonter depuis cette date et atteindre 24200 en juin 1985 et plus de 26000 fin 1986. Les frontaliers ont été touchés par la diminution des effectifs dans l'industrie genevoise, car plus de $40 \%$ d'entre eux travaillaient vers 1975 dans le secteur secondaire: près du quart est débauché entre 1974 et 1977, ce qui illustre la fragilité de cette situation. Mais suite aux différences salariales, l'emploi à Ge- 

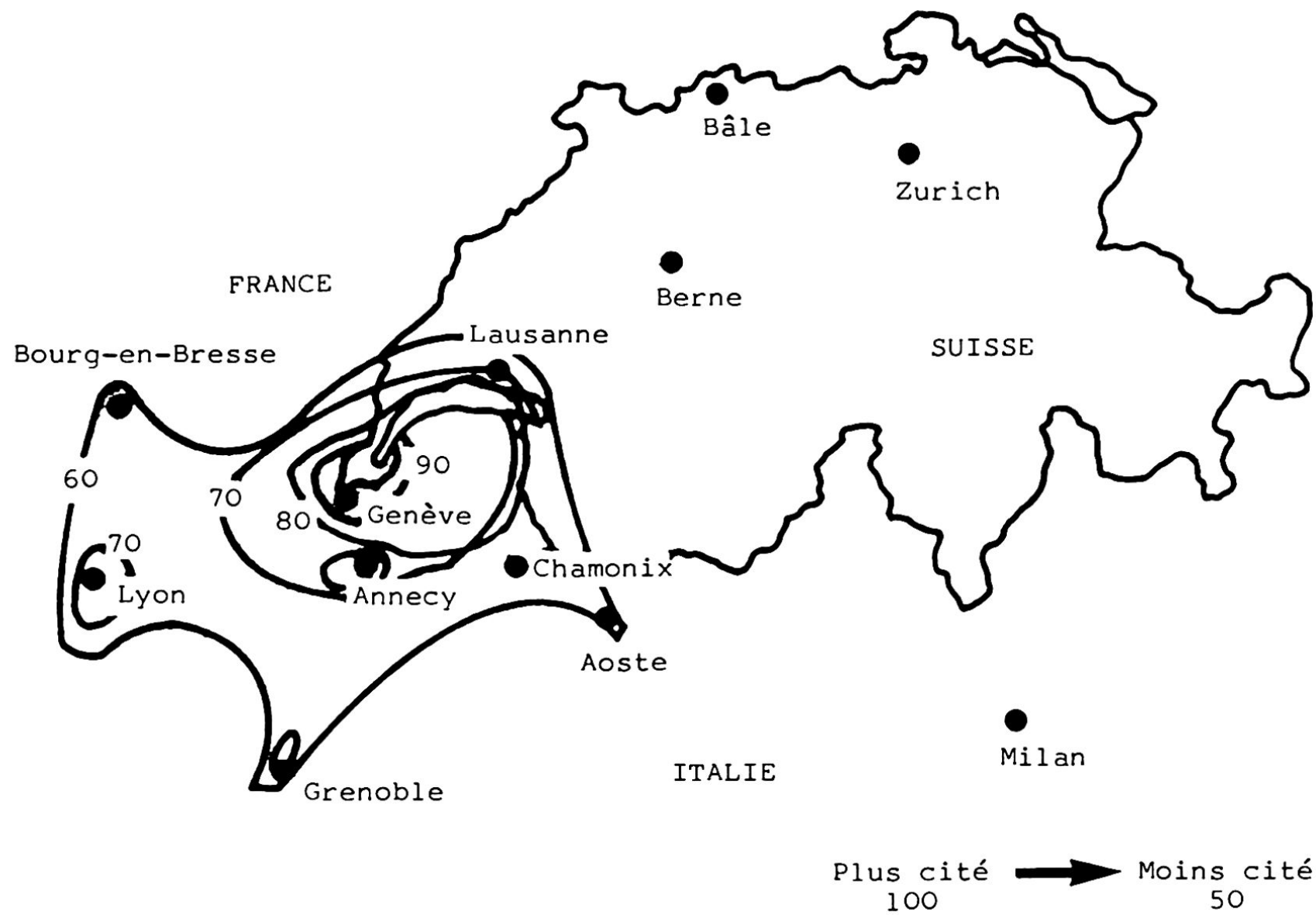

Fig. 2 Carte mentale de frontaliers en provenance du pays de Gex et de Savoie (Enquête: A. Bailly, 1986)

nève attire de plus en plus de Français résidant dans la zone frontière, ce qui explique la reprise des années 80. Vivre en France, avec un salaire suisse et en cotisant, grâce à son conjoint, aux assurances sociales françaises, constitue un avantage économique très net! Le mitage résidentiel pavillonnaire est l'une des conséquences principales, les frontaliers pouvant bâtir des villas dans les zones proches de la frontière, ce qui n'est pas sans conséquence sur les problèmes d'équipement de ces communes. Mais comme les frontaliers dépensent le $90 \%$ de leurs revenus en France, l'incidence sur le développement des commerces et des services est majeure, ce qui explique en partie (avec les achats des Suisses en France) l'excellente infrastructure dont bénéficient les villes et les villages du Pays de Gex et de HauteSavoie. Suite à l'accord franco-suisse du 29 janvier 1973 , premier dans le genre (et qui va servir de modèle), la Suisse accepte le principe de la péréquation financière transfrontalière pour aider les communes françaises dans leur équipement. L'idée, soulignée en introduction, d'un partage des impôts entre le pays d'emploi (qui prélève les impôts des frontaliers à la source) et le pays de résidence est fondamentale pour la coopération transfrontalière. La difficulté majeure dans la négociation provenait du fait que les Autorités genevoises ne voulaient pas que les montants versés passent par Paris; puisqu'il s'agit d'un véritable accord régional, il fallait que l'administration fiscale de Genève reverse directement aux administrations des départements limitrophes, Ain et Haute-Savoie, 3,5\% de la masse salariale des frontaliers. Un transfert à Paris aurait fait «capoter» l'accord puisque le Ministère des Finances aurait pu redistribuer à sa guise les fonds. Grâce au compromis obtenu, l'accord a permis des versements de l'ordre de 10 millions de francs suisses en 1974, 20 millions début 1982 , et près de 40 millions en 1985 ; il va d'ailleurs servir de modèle aux institutions européennes, et dans ce sens la région franco-genevoise fait figure de pionnier dans la coopération financière transnationale. 


\subsection{Les fonctionnaires des organisations internationales}

Outre les frontaliers, près de 5000 fonctionnaires des organisations internationales travaillent à Genève et vivent en France; chiffre relativement stable depuis les années 80 . Au total la ville de Genève, qui possède 232 sièges principaux et secondaires d'organisations internationales, dont 14 gouvernementales, compte environ 20000 fonctionnaires internationaux* (soit $10 \%$ de l'emploi du canton). Le quart de cette population internationale vit non pas à Genève, mais dans la région franco-genevoise. Ce sont les employés du CERN (Centre d'Etude en Recherches Nucléaires) qui résident le plus en France (près de $60 \%$ du personnel dans le Pays de Gex); les fonctionnaires de l'Office des NationsUnies (ONU), du Bureau International du Travail (B.I.T.), de l'Organisation Mondiale de la Santé (O.M.S.), du GATT (Accord Fédéral sur les Tarifs Douaniers et le Commerce), de l'Union Internationale des Télécommunications (U.I.T.), se dispersent aussi dans toute la région en fonction de leur statut socio-économique: les cadres résident dans les quartiers à niveau élevé de Genève, alors que les employés habitent plutôt en France. La frontière dans ce cas marque souvent la hiérarchie sociale, les fonctionnaires aux revenus les plus faibles profitant des avantages économiques d'une localisation dans l'Ain et la Haute-Savoie. Cette préférence pour la France et parfois le Canton de Vaud ne fait que s'accroître suite à la pénurie de logements à Genève, et à leur prix. Les désexternalités économiques de la métropole influencent cette dispersion. Genève joue ainsi le rôle de véritable pôle de développement, qui suivant la théorie de F. Perroux, diffuse son dynamisme à l'ensemble de sa région.

* En 1982 on comptait un total de 14373 personnes employées dans les organisations internationales à Genève, sans tenir compte des missions, consulats et organisations internationales privées; en 1985 ce chiffre dépasse les 15 000:

Office des Nations-Unies, 3550 employés

Bureau International du Travail, 1383

Office Mondial de la Santé, 1279

Union Internationale des Télécommunications, 692

Organisation Météorologique Mondiale, 283

Organisation Mondiale de la Propriété Intellectuelle, 272

Accord Général sur les Tarifs Douaniers et le Commerce, 316

Organisation Européenne pour la Recherche Nucléaire, 3811

Association Européenne de Libre Echange, 74

Union Interparlementaire, Comité International pour les

Migrations, Bureau International d'Education,...

\subsection{Une véritable aire d'emploi transfrontalière}

Devant la montée de ces phénomènes, C. RICQ (Institut Universitaire d'Etudes Européennes, 1979), parlait de «bassin transfrontalier d'emploi» et il élabore en 1983 une carte très significative (fig. no 3), à partir des données du recensement fédéral suisse de 1980 et du recensement français de 1982. Un bassin d'emploi s'est créé malgré la frontière, malgré les systèmes économiques et sociaux différents. Avec les travailleurs en provenance du Canton de Vaud (8374 en 1980), avec les frontaliers et les fonctionnaires internationaux, plus de 29000 personnes allaient travailler à Genève en 1982. Ce chiffre dépasse officiellement les 32000 actuellement, sans compter les frontaliers «au noir», car dans ce domaine les autorisations suivent la réalité avec un certain déclage $(75 \%$ des demandes de permis pour frontaliers ont été agrées en 1986).

Cette insertion de main d'œuvre résidant en France dans les entreprises genevoises se fait dans des secteurs particuliers de l'économie; nous avons déjà vu que plus de $40 \%$ des frontaliers travaillaient en 1974 dans le secteur secondaire. Malgré une baisse de l'emploi industriel, cette part reste de l'ordre de $37 \%$ en 1985 (contre environ $20 \%$ de la population active genevoise dans le secondaire). Plus de $60 \%$ de frontaliers travaillent donc dans le secteur tertiaire, avec des emplois de bon niveau socio-économique: cadres $(20 \%)$, employés $(52 \%)$ et ouvriers spécialisés $(24 \%)$, ce qui explique le niveau de vie assez élevé de cette population. La différence est

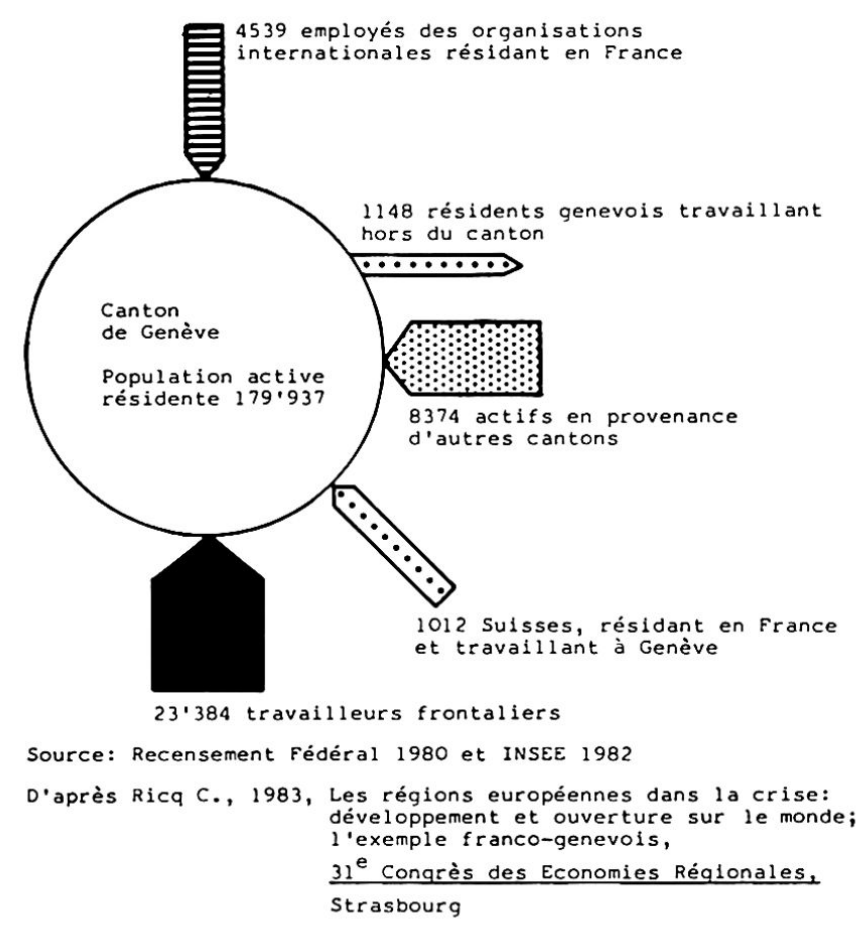

Fig. 3 Migrations de travail dans la Regio Genevensis (1980-1982) 
nette avec beaucoup d'autres régions où les frontaliers occupent essentiellement des emplois non qualifiés. A Genève ce sont les travailleurs saisonniers non français qui occupent ces postes de travail, en particulier dans la construction et l'hôtellerie, sans parler des ateliers.

Si ces phénomènes migratoires marquent profondément la vie économique genevoise en permettant la croissance de la population active, ils influencent aussi, nous l'avons vu, l'économie des régions françaises frontalières, qui bénéficient de flux financiers importants. Ce rôle de Genève est renforcé par les pratiques transfrontalières de l'ensemble de la population: recherche de terrains agricoles, de résidences secondaires et principales, d'aires de loisirs en France. Les conséquences sur les valeurs foncières ne sont pas négligeables; en effet, la zone franche constitue légalement une extension de la zone maraîchère genevoise. Elle permet aussi une diffusion de l'aire de croissance urbaine; même si les Suisses ne peuvent plus habiter légalement en résidence principale dans la région frontière, ils y acquièrent des résidences secondaires. Pour la région française proche, P. GUICHONNET (1975) comptait près de 7000 propriétés (bâties et non bâties) appartenant à des résidents genevois et couvrant $70 \mathrm{~km}^{2}$, soit l'équivalent de $25 \%$ de la surface du Canton. Cette croissance urbaine, au-delà de la frontière, résulte de la petitesse de son territoire, du refus d'extension des zones à urbaniser à l'intérieur du canton, et des différences internationales dans les valeurs foncières et immobilières. La spécialisation spatiale des activités entre les deux régions, de part et d'autre de la frontière, provient de leurs avantages comparatifs. A Genève l'emploi, l'urbanisation dense, à l'aire régionale française l'agriculture, les résidences secondaires, l'urbanisation diffuse. On peut parler de dépendance, mais aussi de complémentarité dans ces phénomènes; une complémentarité malheureusement encore mal mise en valeur par les Autorités dans leurs politiques régionales. En effet, les doublets d'infrastructure sont tout aussi nombreux que les règlements tâtillons gênant l'implantation d'activités et le travail des personnes de part et d'autre de la frontière. C'est grâce aux pratiques et au vécu des gens, plus qu'aux institutions, que la Regio Genevensis se constitue.

\section{Des activités transfrontalières et internationales}

Non seulement une partie importante de la population active vient de l'extérieur du canton, mais les entreprises sont ouvertes sur l'extérieur. Rappelons à ce sujet que le commerce extérieur de Genève, pour le premier trimestre 1985, représentait 1396,4 millions de francs.

Pour mesurer cette ouverture, il nous faut évaluer à la fois la dépendance économique et son pouvoir de contrôle. En 1980, 8,9\% des entreprises, regroupant $34 \%$ des emplois du canton dépendent de l'extérieur. Il s'agit essentiellement de grosses entreprises privées ( $23 \%$ de l'emploi), des organisations internationales $(7,7 \%)$ et du secteur fédéral $(3,1 \%)$ (fig. no 4), comptant au total près de $68000 \mathrm{em}$ plois, ce qui illustre l'attraction exercée par Genève sur le monde extérieur.

Ainsi peut-on expliquer qu'en 1954 le CERN ait décidé d'implanter son laboratoire européen d'étude de physique des particules au Nord-Ouest de Genève sur des terrains ( $40 \mathrm{ha}$ ) mis à disposition par la Confédération, auxquels allaient s'ajouter des terrains donnés par la France. Un premier accélérateur de particules ( $7 \mathrm{~km}$ de diamètre) en 1971, est suivi d'un deuxième plus grand (L.E.P. de $27 \mathrm{~km}$ de diamètre) dont les travaux sont commencés en 1982 sur territoires français et suisse. Outre le renom apporté par ce centre, les retombées économiques sont importantes: près de $38 \%$ du budget du CERN, soit 750 millions de francs suisses, est dépensé en Suisse (masse salariale, travaux, achat de matériel) et un pourcentage semblable en France, alors que la contribution de ces deux pays au budget du CERN est respectivement de $4 \%$ et $22 \%$ ). Ce centre crée en outre un véritable noyau technologique favorisant l'essor de P.M.E. régionales et l'attrait d'entreprises internationales. Les communes du Pays de Gex ont compris ces potentialités en développant, avec l'aide d'une société anglaise, dans la région de Thoiry, à proximité du CERN, un ambitieux projet de parc de haute technologie qui se propose d'accueillir des unités de production de prototypes ou de micro-objets ainsi que des services aux entreprises. Ce projet complète le Technoparc Gessien installé à St-Genis qui constitue une zone industrielle plus classique. Nous assistons donc à une diffusion transfrontalière de l'influence du CERN, qui montre clairement le caractère international du développement économique de la Regio Genevensis. L'examen de la provenance internationale des flux d'investissement à Genève confirme l'attrait international du canton: les Etats-Unis représentent $49,3 \%$ de ces flux devant la France $18,9 \%$. Ces liens économiques et culturels montrent une volonté des entreprises américaines d'accéder au marché européen, et des entreprises françaises d'accéder au marché helvétique à partir d'une ville prestigieuse. L'image de Genève joue un rôle positif dans cet attrait d'entreprises étrangères: le canton en bénéficie, tout comme l'ensemble de la région, qui profite de ce dynamisme économique.

Les entreprises genevoises ayant leur siège dans le canton contrôlent également plus de 10000 emplois en Suisse. Par des participations financières majoritaires et minoritaires, ces entreprises étendent leur influence à l'étranger, par exemple (CERJAT. 1983) dans la région Rhône-Alpes. «Ville-Etat, coupée de son arrière-pays, Genève a pu bénéficier dès l'épo- 


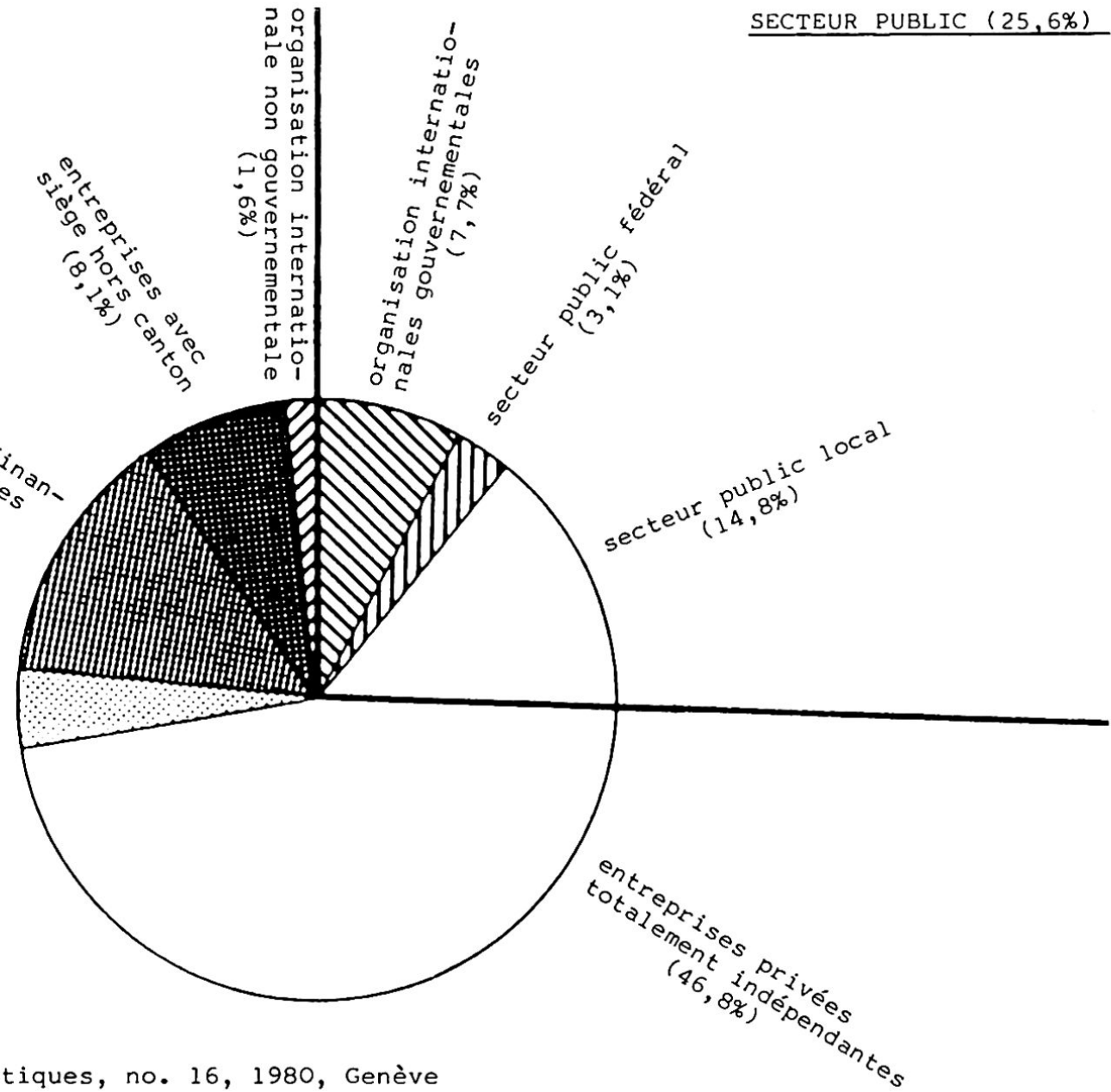

Source: Aspects Statistiques, no. 16, 1980, Genève

Fig. 4 Emplois du canton selon le type de dépendance des entreprises

que féodale de franchises locales lui permettant de s'approvisionner et de se désenclaver» (JEANNERET. 1985 , p. 136). Ces participations se situent de manière privilégiée dans deux espaces géographiques, la zone franche et les départements français proches.

Reconnues au Congrès de Vienne (1815), malgré une tentative française de les supprimer (1923), les zones franches possèdent un régime de franchise complexe qui permet les importations de France vers la Suisse avec des taxes fiscales réduites (et de la Suisse vers la zone franche, mais pas vers la France). Prenant de plus en plus d'importance sur le plan agricole, ceinture maraîchère de la ville, les importations vers Genève ont quintuplé depuis 1960 pour représenter plus de $1 \%$ des flux entrant dans le canton. Mais le développement industriel genevois ne s'est pas encore transmis dans toutes les zones franches, qui restent avant tout résidentielles et agricoles. Les échanges, de type campagne-ville, restent dissymétriques: exportation de matières premières aux prix suisses vers Genève, résidence de frontaliers travaillant à Genève, mais encore peu de créations d'emplois industriels et de services. Les entreprises, lorsqu'elles sont financées par des capitaux genevois, se localisent un peu plus loin dans les départements français de la région Rhône-Alpes.

La campagne French Geneva County lancée en Californie par l'Agence Economique Départementale de la Haute-Savoie pour attirer des industriels étrangers dans la région frontière va-t-elle, tout comme la Technopolis de Thoiry, modifier cette tendance? En utilisant à la fois le nom de Genève, du Mont-Blanc et une liste des industries de pointe du département sur la publicité, l'Agence joue sur une double image: celle d'une ville de prestige dans ce qui est qualifié d'Europe's prosperity belt, et celle de la montagne dans la Snow-Belt alpine.

La zone industrielle d'Archamps, au pied du Salève, qualifiée parfois de Silicon Valley savoyarde, devrait bénéficier de cette campagne publicitaire. En 
profitant de la présence de l'Autoroute 42 (autoroute Blanche vers l'Italie et Paris), de la jonction avec les autoroutes suisses, de l'accès à l'aéroport de Cointrin et à sa gare ferroviaire, cette zone donnera-t-elle le signal de l'industrialisation de certains secteurs des zones franches? A la fois porte de la Communauté Economique Européenne pour la Suisse, et porte de la Suisse pour les industriels français, elle bénéficie d'un double avantage transfrontalier. Il faudra attendre une dizaine d'années pour évaluer les résultats de cette tentative.

En attendant regardons, à travers les analyses de JEANNERET et MAILLAT (1981), le rôle des participations étrangères dans les régions françaises frontières avec la Suisse. Douze des vingt-trois participations helvétiques en Haute-Savoie sont originaires du canton de Genève. Il s'agit d'une influence par proximité, qui explique que la part totale des investissements helvétiques par rapport aux participations étrangères dépasse $25 \%$ dans la région RhôneAlpes. La motivation principale de ces investissements devient de plus en plus l'accès aux marchés de la Communauté Economique Européenne, tout en étant dans des villes ou régions aisément accessibles depuis Genève.

Les exportations du canton à destination des départements français voisins reflètent l'importance de cette influence helvétique. Sans tenir compte des exportations aéroportuaires, en $198228,8 \%$ de la valeur des exportations genevoises vers la France était destiné aux départements proches. La HauteSavoie constitue un exemple majeur du rôle de l'accessibilité sur la vie économique.

Ainsi, malgré le protectionnisme suisse, fondé sur le concept de neutralité, Genève poursuit depuis longtemps une politique économique extérieure où le libéralisme provient de l'intérêt international. De ce caractère international résulte une dépendance, en particulier des Etats-Unis et de pays européens. C'est à la fois un inconvénient et un avantage; inconvénient car le contrôle est difficile, avantage car l'image positive de Genève permet d'attirer de nouvelles entreprises créatrices d'emplois et de développer un secteur de service international puissant. Le récent projet d'encouragement et de financement de l'innovation que devrait permettre la mise en place d'une fondation destinée à aider les entreprises, a comme but de favoriser la croissance d'activités de pointe dans le canton. Un fonds d'Evaluation et d'Assistance pour aider à l'évaluation de projets, un Fonds de Développement pour assister des entreprises en croissance rapide, et un Fonds de Financement «capital-innovation» pour aider les entreprises nouvelles devraient contribuer au renouvellement du tissu industriel genevois. L'ensemble de l'économie peut profiter de la croissance de nouvelles activités motrices. Les services bancaires, l'hôtellerie (qui ne reçoit que $15 \%$ d'hôtes suisses en moyenne ces dernières années) en sont les grands bénéficiaires, tout comme les activités commerciales. Genève possède en effet des équipements commerciaux et de services surdimensionnés par rapport à sa population, utilisés à la fois par la clientèle régionale mais aussi par une clientèle internationale à fort pouvoir d'achat. L'aéroport de Cointrin, avec ses secteurs français et suisse, ne se contente pas de servir les voyageurs résidant à Genève, dans l'Ain et la Haute-Savoie, mais devient de plus en plus une porte favorisant les activités internationales et le tourisme. Il n'est qu'à constater l'augmentation du nombre de passages les mois d'hiver pour voir que des milliers de skieurs utilisent Cointrin pour se rendre ensuite dans les stations de ski de Savoie: 413900 passagers en mars 1985 dont 115000 par vols spéciaux (par comparaison, en période non touristique comme novembre, ce nombre se réduit à 307000 passagers dont 13500 par vols spéciaux). La desserte en T.G.V. de la gare Cornavin renforce ce rôle régional de Genève pour l'accueil de personnes se rendant dans les zones voisines malgré la concurrence naissante de Bellegarde qui a aménagé une gare routière pratique pour desservir les stations alpines.

\section{Doubles emplois et difficultés de fonctionnement}

Malgré cette centralité incontestable de Genève, la présence de régions placées dans des systèmes institutionnels différents entraîne de nombreux doubles emplois; par exemple dans les secteurs hospitaliers, éducatifs, administratifs. La coordination inter-régionale n'est pas satisfaisante: ainsi, par suite de l'absence d'équivalences claires, les étudiants français vont à l'Université à Lyon ou à Grenoble; suite aussi aux difficultés de prise en charge par la Sécurité Sociale des frais médicaux et au manque d'accord comptable général entre cet organisme et l'Hôpital Cantonal, celui-ci ne peut jouer pleinement son rôle régional. Malgré l'accord de coopération de février 1984 qui prévoit d'appliquer le tarif des Confédérés aux frontaliers et à leurs familles, malgré l'accord de juillet 1985 entre l'Hôpital Cantonal et trois caisses-maladie françaises qui règlent directement les frais de leurs assurés pour une durée de 30 jours, les chiffres de fréquentation des hôpitaux genevois par les frontaliers restent dérisoires: de juillet à décembre 1985, 90 patients, et 148 au premier semestre 1986 ! Il faut dire qu'au-delà de trente jours les frontaliers ne bénéficient plus du tarif des Confédérés, mais de celui des étrangers domiciliés hors de Suisse. Ni les médecins hospitaliers français, ni la Sécurité Sociale ne tiennent à cet accord qui favoriserait l'Hôpital Cantonal. D'ailleurs nombre de cas traités par Genève sont des urgences; pour les autres cas graves le système hospitalier français est utilisé avec déplacement des patients sur Lyon, Grenoble... Même le système bancaire fonctionne dans 
chaque pays suivant les normes nationales; et les douaniers veillent au grain lorsque le contrôle des changes est renforcé pour éviter les flux financiers internationaux. La liste de ces tracas et entraves pourrait être plus longue; elle illustre l'ignorance des Etats nationaux, face aux problèmes transfrontaliers. Le conflit franco-suisse en ce qui concerne les radios locales transfrontalières en constitue une illustration. Une dizaine de stations privées françaises émettent dans le bassin lémanique à des puissances jugées exagérées; citons par exemple Radio Thollon-Les Mémises diffusant depuis les hauts de Thonon et Radio Plus depuis Annemasse. L'Union Internationale des Télécommunications vient de rappeler à l'administration française son obligation internationale de partage équitable des fréquences et la cour d'appel de Chambéry a condamné Radio Thollon-Les Mémises à une amende et à la confiscation du matériel. Mais cette radio s'est pourvue en cassation et les émissions se poursuivent; le conflit aussi. Ce ne sont pas les autorisations d'installation d'émetteurs privés helvétiques en France, au Salève, qui résoudront le problème. Dans ce domaine, comme dans d'autres, des accords précis doivent être signés et respectés.

Tout comme pour les radios françaises, l'utilisation, par certains commerces, des avantages de coûts en France, pour profiter d'un marché suisse, est particulièrement bénéfique... A la zone française proche, les commerces alimentaires, de sport, de vêtements fournissent des produits à des prix inférieurs à ceux pratiqués à Genève; dans cette ville, les commerces de luxe et de haut niveau sont destinés à la clientèle internationale. Apparaît ainsi une spécialisation fonctionnelle, variable dans le temps, fondée sur l'effet frontière; en résulte une certaine fragilité puisque l'avantage comparatif est en général lié aux systèmes de taxation différents. Tout blocage peut remettre en cause ce système.

\section{Les structures transfrontalières: les progrès en cours}

Image transfrontalière, migrations transfrontalières, flux économiques transfrontaliers, tous ces éléments contribuent à la mise en place de structures destinées à réduire les contraintes des Etats nationaux. Nous avons déjà présenté l'accord francosuisse (29 janvier 1973) de péréquation fiscale transfrontalière; mais depuis cet accord pionnier, petit à petit, des institutions transfrontalières ont été créées. L'espace économique ne coïncidant plus avec l'espace national, il est devenu impératif, malgré les réticences des institutions centralisées françaises, de limiter les perturbations dans le flux de biens et de services liées à l'effet frontière.

La notion de Regio Genevensis repose à la fois sur un mode de fonctionnement et une image ancrée dans l'esprit de la population. Reste donc à en tirer des leçons sur le plan légal, ce qui est toujours long. Rappelons donc les grandes étapes de la mise en place des institutions transfrontalières, réponse à une situation de fait:

1881 Décision de raccordement du chemin de fer de Haute-Savoie à Genève-Eaux-Vives

1935 Convention sur le régime fiscal des frontaliers

1946 Convention sur la circulation frontalière

1956 Accord pour la création de l'aéroport international de Genève-Cointrin

1973 Accord de compensation fiscale transfrontalier et approbation de la constitution d'une Commission consultative mixte transfrontalière traitant des questions franco-genevoises

1974 Création du Comité Régional Franco-Genevois

1982 Création de la Communauté de Travail des Alpes Occidentales (Genève-Vaud-Valais-région Rhône-Alpes, Provence-Côte d'AzurPiémont-Lombardie)

1986 Création du Conseil du Léman (Genève-Vaud-Valais-Rhône-Alpes)

Suite à la création de la Commission consultative mixte (12 juillet 1974) et du Comité Régional Franco-Genevois (25 mars 1974), vont être établies quatre grandes Commissions permanentes destinées à traiter des questions de voisinage entre les départements de l'Ain, de la Haute-Savoie et le Canton de Genève (fig. no 5). Ces commissions ont pour tâche la résolution des problèmes posés par la "population frontalière», «l'environnement et l'aménagement rural et urbain», «l'économie et les transports» et «la culture et les loisirs». Malgré les difficultés d'articulation entre problèmes nationaux et régionaux, les institutions transfrontalières ont permis de régler quelques questions de fonctionnement. Prenons comme exemples les raccordements de communes françaises (Viry et Valleiry) à la station d'épuration suisse de Chancy et, inversément, dans le futur, celui de la commune suisse de Veigy sur la station française d'Anières. La question de qualité des eaux et de lutte contre les pollutions est d'ailleurs un domaine privilégié de coopération transfrontalière: étude des bassins de trente rivières pour construire des stations d'épuration, travaux de la Commission Internationale pour la Protection des Eaux du Léman permettant en particulier la coordination lors de pollutions par les hydrocarbures (systèmes de détection et de luttes communs). Dans le domaine nucléaire, Genève est en liaison directe avec les centrales du Bugey et de Crey-Malville, pour pouvoir éventuellement déclencher le Plan de Secours en cas de catastrophe en temps de paix.

Sur d'autres plans signalons la reconnaissance des diplômes d'apprentissage genevois par la France, les échanges scolaires de classes primaires genevoi- 


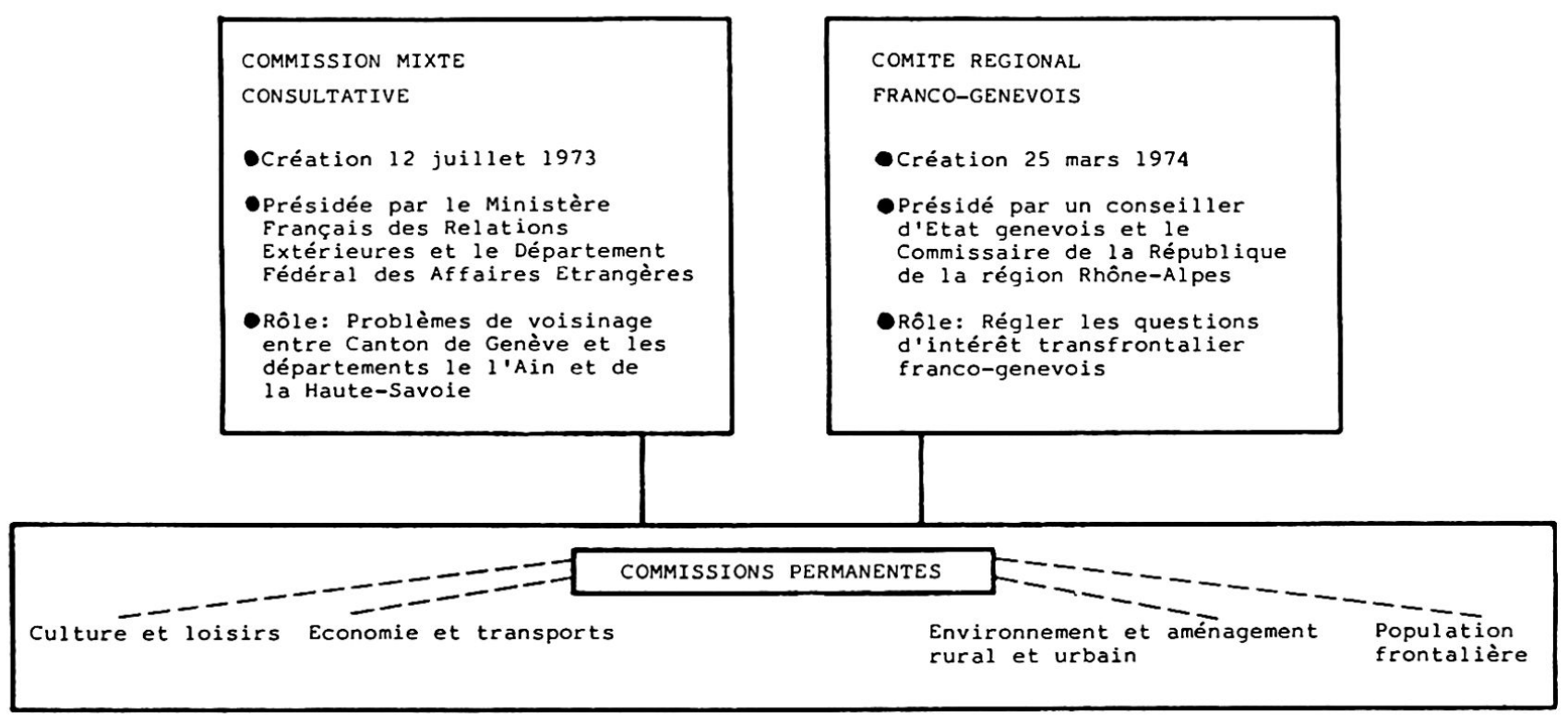

Fig. 5 Institutions transfrontalières franco-genevoises depuis 1973

ses en Haute-Savoie; mais il n'y a toujours pas d'équivalence entre maturité suisse et baccalauréat, ni reconnaissance des diplômes universitaires. Mentionnons aussi l'analyse comparative des Plans d'Occupation des Sols français et des Plans d'Aménagement suisse pour mieux coordonner zones de logements, voies de communication et zones d'emploi. Un inventaire des problèmes peut être dressé, facilitant quelques réalisations communes: desserte par les transports en commun (bus et trains)* des communes françaises et genevoises, coordination des travaux routiers et autoroutiers, accord pour la construction d'infrastructures touristiques (téléphérique du Salève), expositions itinérantes...

Devant l'ampleur des problèmes à résoudre, nombre d'associations transfrontalières se sont créées. Sans vouloir dresser une liste exhaustive, signalons le Groupement des Frontaliers de la Haute-Savoie, créé en 1963 pour défendre les intérêts des travailleurs frontaliers, le Groupe de Concertation des Cantons frontaliers limitrophes de la France, mis en place en 1982 pour harmoniser les politiques régionales, l'Association Genevoise pour le Développement des Relations Interrégionales (Genève, Vaud, Valais, Ain, Haute-Savoie) et la Coordination Economique et Sociale, regroupant les syndicats gene-

* Signalons en particulier en 1985 l'accord d'électrification de la ligne Annemasse-Genève et la vente d'abonnements combinés entre la Société Nationale des Chemins de Fer Français et les Transports Publics Genevois. vois, vaudois et français. Mais bien des problèmes restent en suspens, tel celui d'une meilleure utilisation de l'Hôpital Cantonal par les assurés sociaux français, de l'aménagement d'espaces de loisirs, des zones industrielles, des extensions résidentielles de Genève, des radios locales...

Du fait des possibilités décisionnelles limitées des institutions transfrontalières, en particulier en France, bien des pouvoirs restent concentrés à Paris. Les lois françaises du 2 mars 1982 et du 8 janvier 1983 ne permettent pas de répondre pleinement aux solidarités régionales qui se crééent. Les obstacles restent nombreux: règlements du Marché Commun et de l'Association Européenne de Libre Echange, dont fait partie la Suisse, droits et systèmes fiscaux différents...

Signalons aussi que tous les problèmes de coopération transfrontalière ne sont pas transnationaux. Ainsi le dialogue est-il juste amorcé entre le Canton de Vaud et celui de Genève, malgré l'exurbanisation de travailleurs genevois en terre vaudoise. Des délégations de conseillers d'Etat se rencontrent, mais les réalisations communes sont faibles: on parle des zones d'habitat des salariés genevois dans la région frontalière, de l'imposition des personnes dont les lieux de travail et d'habitat sont différents: Vaud serait d'accord pour contribuer à des projets genevois d'intérêt commun, par exemple dans les transports publics, pour résoudre les problèmes des déplacements pendulaires... Malgré la participation des deux cantons au Comité de travail des Alpes Occidentales, les réalisations sont plus faibles que dans la coopération entre Genève et la France. Là aussi 
la réalité des pratiques spatiales précède les accords entre cantons qui n'ont pas pris l'habitude de traiter ensemble des problèmes communs.

\section{Conclusions}

Le Regio Genevensis existe; nul ne peut le contester, même si l'image mentale des habitants varie légèrement en fonction du pays de résidence, car elle correspond à des pratiques et à un vécu collectif. Une ville d'importance internationale, véritable pôle de croissance économique, dynamise sa région proche et lointaine; et en utilisant les avantages comparatifs de deux pays différents, les entreprises de services, industrielles, commerciales et agricoles bénéficient des attraits complémentaires de cette région transfrontalière.

Mais à cet espace vécu se superposent deux espaces nationaux, qui tendent à freiner le mouvement du secteur privé et des individus. Malgré des progrès, surtout depuis 1973, la dynamique institutionnelle reste en retard sur les faits et parfois on assiste à un véritable enlisement administratif défavorable au développement de l'entité régionale. Les interventions administratives, à des échelles géographiques différentes, celles de la nation et de la région, répondent à des logiques différentes; et souvent les régions en subissent les conséquences puisque les décisions n'ont pas été imaginées pour ces cas marginaux. L'intérêt national ne correspond pas toujours à celui des régions. L'exemple récent de l'introduction des visas pour pénétrer en France en constitue l'illustration: si les Suisses et les habitants des pays de la Communauté Economique Européenne sont dispensés du visa, il n'en va pas de même pour les autres nations. Or plus de 50000 ressortissants de ces pays vivent à Genève... et se déplacent souvent en France! Files d'attente au Consulat à Genève et à la frontière en sont la conséquence, négative pour le devenir de cette région transfrontalière. Un pas en arrière seulement, car la région continue à fonctionner.

De nouveaux modes d'intervention territoriaux se mettent en place dans les régions transfrontalières; progressivement les gouvernements locaux développent des politiques transnationales pour régler les problèmes concrets liés aux pratiques territoriales. On peut parler de véritable politique étrangère cantonale.

L'évolution de la Regio Genevensis suppose qu'aux problèmes communs qui se posent soient trouvées des solutions communes, dans le respect des caractères de chaque entité. C'est par la mise en valeur des avantages complémentaires, centralité de Genève, disponibilité foncière de la France, que pourra se produire un rééquilibrage économique, évitant les dépendances ville-banlieue.
Ce mouvement ne sera possible que si les collectivités locales bénéficient de pouvoirs décisionnels larges et de responsabilités financières pour pouvoir gérer la région transfrontalière; car comment une région déchirée administrativement peut-elle, sans ces moyens, lutter face à des entreprises et des problèmes qui se posent de plus en plus à l'échelle internationale?

La mise en place de politique transnationales reste lente. Les raisons sont en partie liées aux échelles géographiques d'intervention des deux unités (fig. no 6): pour la France la Regio Genevensis ne constitue qu'un segment de frontière, alors que pour le Canton de Genève elle couvre toute la frontière avec l'étranger. Les intérêts nationaux et locaux n'étant pas nécessairement en adéquation, des divergences peuvent apparaître, entravant l'élaboration de mesures locales cohérentes. Le deuxième raison de la lenteur dans la création d'une véritable Regio Genevensis provient de la multiplicité des acteurs, dont deux, la région et le canton, ne sont pas souverains. La complexité des systèmes relationnels, malgré la création de comités régionaux transfrontaliers, explique une forme d'impuissance face au processus d'internationalisation. Seule une véritable géo-politique régionale, sous la responsabilité de la région et du canton, pourra intégrer les valeurs des populations locales et de leurs pratiques.

\section{Résumé}

Pionnière en Europe depuis la création en 1973 de la Commission consultative mixte transfrontalière et en 1974 du Comité Régional Franco-Genevois, la Regio Genevensis a su établir des séries de mesures transfrontalières, dont la plus connue est la compensation fiscale. Bien souvent la région sert d'exemple dans les relations transfrontalières; position envia-

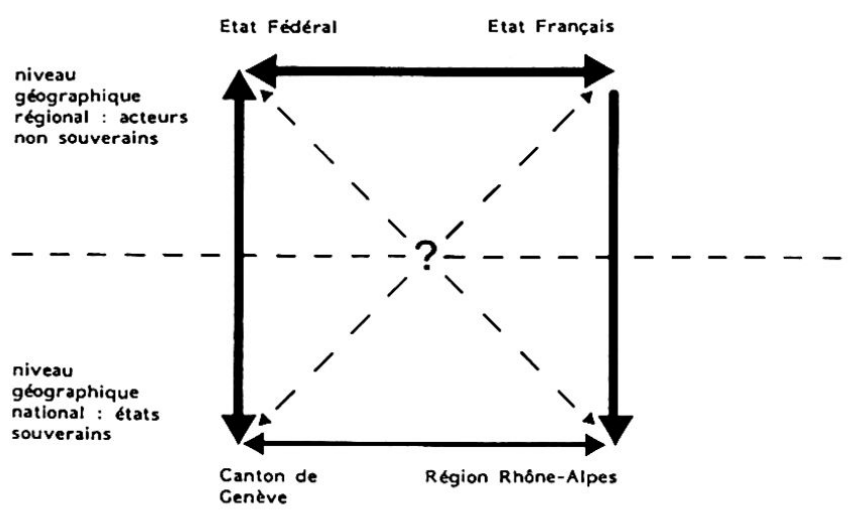

Fig. 6 Le rôle des acteurs politiques de la région franco-genevoise 
ble, mais aussi difficile, car l'engagement international d'un canton non souverain face à un Etat centralisé résulte de compromis délicats à élaborer et pourtant nécessaires au vu des images mentales et des pratiques transfrontalières des populations.

\section{Abstract}

The Franco-Geneva region has been a pioneer region since the establishment of a "Transborder Commission" in 1973 which deals with international problems. One of the most important results of this international cooperation was a financial agreement.

$3.5 \%$ of the wages earned by the French frontaliers working in the Canton of Geneva are redistributed to French regional authorities. The total amount which was 10 million Swiss francs (for 25000 workers) in 1974 has risen to 40 million Swiss francs at present. It allows local communities to finance infrastructures and services for the resident population.

This agreement is a model case in Europe. However, not all the problems related to the presence of a national boundary dividing an economic region and a labour market are solved. The French side of the region is often regarded as a peripheral area for Geneva. As its agricultural belt and recreational zone with secondary homes, skiing areas and regional parks, it offers services to the overcrowded center. Owing to the centralization, however, the French population does not benefit from all the services offered by the government of Geneva. France has its own systems of education, public health and administration. Even if people live and act in this regional entity, administrative barriers are still in effect. Solving common problems are one of the main objectives of local authorities in order to create a real Regio Genevensis. While the private sector can deal with local adavantages on each side of the bor- der with relative ease, it takes longer for the "national system" to react to the evolutionary trend toward a closer cooperation.

\section{Bibliographie}

ASPECTS STATISTIQUES (1975 à 1985): Service Cantonal de la Statistique de Genève.

BAETTIG, M. (1986): Genève doit-elle rester suisse? Favre, Lausanne.

BAILLY, A. et BEGUIN, H. (1982): Introduction à la géographie humaine. Masson, Paris.

BUFFAT, F. (1979): Les zones franches de la région franco-genevoise à l'heure de l'intégration européenne. Institut Universitaire d'Etudes Euorpéennes, Genève.

CERJAT, B. (1983): La contribution de la Suisse à l'industrie française. In: Revue Economique Franco-Suisse, no 4.

ENTREPRISE ROMANDE (1986): Numéro Spécial 2026, 19 décembre.

INSTITUT UNIVERSITAIRE D'ÉTUDES EUROPÉENNES (1979): Genève et l'Europe: un exemple de coopération régionale transfrontalière. Genève.

LE GLOBE (1081): Genève, Aménagement d'un espace urbain. Numéro Spécial, 121.

JAGGI, Y. (1983): Politique économique extérieure, défense nationale économique. Presses Polytechniques Romandes, Lausanne.

JEANNERET, P. (1985): Régions et frontières internationales. EDES, Neuchâtel.

JEANNERET, P. et MAILLAT, D. (1981): Jura, canton frontière. G.E.E., Neuchâtel.

RAFFESTIN, C./GUICHONNET, P. et HUSSY, C. (1975): Frontières et Sociétés: le cas franco-genevois. L'Age d'Homme, Lausanne.

RICQ C. (1983): Les régions européennes dans la crise: développement et ouverture sur le monde; l'exemple francogenevois. 31e Congrès des Economies Régionales, Strasbourg.

VERNEX, J.-C. (1981): Genève: représentations d'une ville. In: Le Globe, 121, p. 241-250. 